\title{
Positive Streamer Discharges along Liquid Dielectric Surfaces: Effect of Dielectric Constant and Surface Properties
}

\author{
M. Akyuz, P. P. Cortet and V. Cooray \\ Division for Electricity and Lightning Research \\ Ångström Laboratory \\ Uppsala University, Sweden
}

\begin{abstract}
This paper presents investigations on positive streamer discharges propagating in air along the surface of two non-mixing dielectric liquids. By measuring the currents associated with the streamer discharge propagating along the surface of the two non-mixing liquids for varying ratio of their volume, we analyzed the effect of the dielectric constant (the capacitive effect) and the surface properties. Especially, we separated and quantified the magnitude of each contribution. Two different combinations were studied: silicone oil together with glycerol and transformer oil together with glycerol. For these material combinations, we found that the influence of the dielectric constant is about 3 times larger than the effect of the surface properties. Further, by using a simple model of the streamer discharge, we determined with a 3D field calculation program the capacitive behavior of the two nonmixing dielectric materials for varying ratio of their volume. We found that, in order to obtain the observed capacitive dependence, the streamer must propagate literally on the insulator surface.
\end{abstract}

Index Terms - Streamer discharge, dielectric liquid mixtures, silicone oil, glycerol, transformer oil, dielectric constant, surface properties.

\section{INTRODUCTION}

$\mathrm{T}_{\mathrm{n}}$ HE increased use of electronic devices in our modern society implies a higher demand on the quality of the electric power supply. In many practical situations, it is found that the quality of the electric power is determined by the performance of the insulating elements used in the power generation and transmission utilities. The traditional materials used for outdoor insulation have until recently been glass and porcelain. However, insulators made of these materials are heavy, brittle, and complicated to manufacture. In recent years, therefore, there has been a trend to replace these insulators with polymeric materials. These new materials are easier to manufacture and there is a large flexibility in the choice of their properties. A lot of damage is caused by the electrical breakdown over the insulators which can affect their insulating properties. This has led to an increasing demand for the understanding of characteristics of discharges over dielectric surfaces. The results of the research on this topic will contribute to the understanding of interaction between

Manuscript received on 26 November 2002, in final form 26 November 2004. surfaces and discharge processes, especially, the development of pre-discharge phenomena (like streamers) on insulating surfaces. These processes, which always precede the actual flashover, are initiated when the applied voltage in the system is approaching the critical breakdown value.

Streamer discharges have been studied extensively, both theoretically and experimentally. However, the mechanisms governing the characteristics of streamer discharges along an insulator surface are very complex and are still today not solved [1-5]. In our previous study [6], we compared the characteristics of streamer discharges propagating in air and along different insulating surfaces. Clear differences were detected between the investigated materials and a discussion was presented which elucidated some possible mechanisms contributing to the observations. Especially, it was shown that the dielectric constant is one important parameter which will affect the characteristics of the streamer. The other suggested mechanisms were related to the properties of the surface itself, for example the ionization potential of the surface, the effect of charge accumulated or deposited on the surface, etc. However, 
although, the previous work clarified the various possible mechanisms, it was not possible to quantify the individual contributions and therefore it was difficult to conclude in general why the investigated materials differed in their appearance when introduced in the stressed electrode gap. In this report we have continued those previously conducted investigations. Especially, our main goal is to answer the question: How can we separate the effect of the dielectric constant from the surface properties when a streamer is propagating along the surface of an insulator? In this report we present a method to accomplish that by changing the relative volume of two non-mixing liquids and measuring the corresponding streamer currents. Indeed, the method not only separates the dielectric constant effect from the surface properties but also offers a quantification of these two individual contributions.

\section{EXPERIMENTAL DETAILS}

An arrangement with a quasi-uniform electric field distribution between two parallel plane electrodes was constructed for this experiment, same as the one used in our previous studies $[7,8]$. A negative dc voltage $U_{n}$ applied across the two plane electrodes provided a uniform average electric field $\mathrm{E}$, defined as $\mathrm{E}=\mathrm{U}_{\mathrm{n}} / \mathrm{d}$ where $\mathrm{d}=35$ $\mathrm{mm}$ is the gap distance. The two parallel electrodes (made of brass) were $90 \mathrm{~mm}$ in diameter with Rogowski profile. A sharp, insulated, needle was placed at the centre of the anode (grounded electrode) through a $16 \mathrm{~mm}$ diameter hole on it. The needle was subjected to a step impulse voltage of $5 \mu$ s duration and magnitude $3 \mathrm{kV}$ for triggering streamer discharges. The needle length in the gap (i.e., from the tip to the surface of the anode) was $3 \mathrm{~mm}$, and the diameter of the needle was $0.35 \mathrm{~mm}$. In the experiment, the voltage applied on the needle, the currents on the needle and cathode were measured. The optical radiation from streamer discharges also was detected by two optical fibres, one placed close to the needle tip and the other close to the cathode surface. Detailed description of the set-up and the measuring system can be found in [7, 8].

A Plexiglas box (of dimensions $120 \times 35 \times 65 \mathrm{~mm}^{3}$ ) was introduced between the two Rogowski electrodes, see Figure 1. On both sides of the box, at the upper part (the part which is just over the liquid surface), a thin plastic film $(0.3 \mathrm{~mm})$ was placed in order to prevent the liquid from dropping out of the box during the measurements. The plastic film was thin in order not to inhibit the streamer propagation. At one side of the box there was made a small hole which allowed the needle to enter into the box to generate the streamers above the dielectric surface. The box was filled with two non-mixing dielectric liquids to the edge of the Plexiglas. The protruding insulated needle was placed at a height of $3 \mathrm{~mm}$ above the non-mixing dielectric liquids. The reason for choosing this height was to come as close as possible to the uppermost

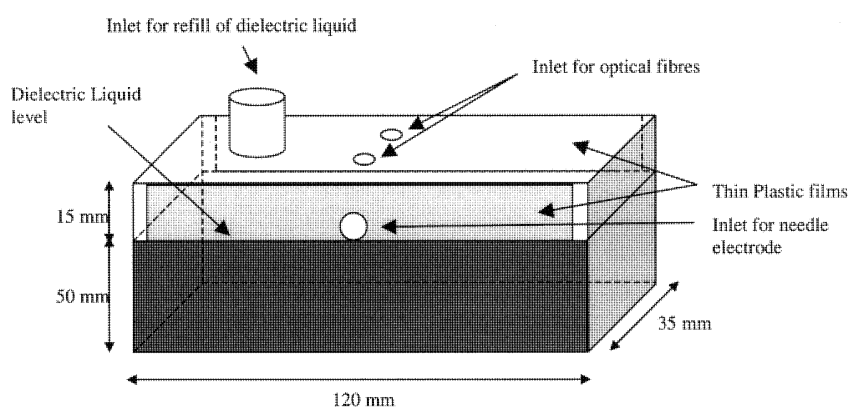

Figure 1. Illustration of the Plexiglas box containing two non-mixing dielectric liquids. The dimensions of the box are also noted in the figure.

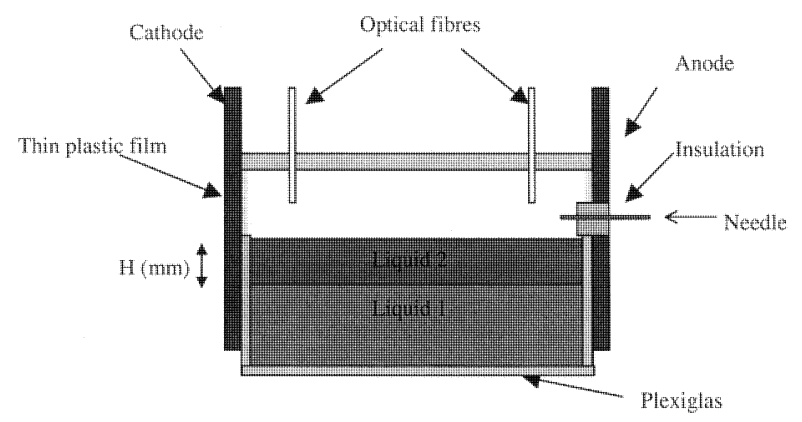

Figure 2. A cross-section of the Plexiglas box placed in-between the plane electrodes of the three-electrode system. The box is filled with the two non-mixing dielectric liquids, liquid 1 and liquid 2 as marked in the figure. $\mathrm{H}$ is the height (thickness) (in $\mathrm{mm}$ ) of the uppermost liquid.

liquid surface but still not being in contact. The total thickness of the two liquids was $48 \mathrm{~mm}$ (excluding the thickness $2 \mathrm{~mm}$ of the Plexiglas box) as illustrated in Figure 1 . The total volume of the two non-mixing liquids was $\approx 172 \mathrm{ml}\left(116 \times 31 \times 48 \mathrm{~mm}^{3}\right)$. It was possible to change the amount of each liquid by a hole placed at the upper side of the box. To achieve this, we used a $1 \mathrm{ml}$ and a $5 \mathrm{ml}$ pipette with a pro-pipette to put and remove certain amounts of liquid. A Plexiglas plate was also fixed to the top of the box in order to mount the optical fibers at right position in the gap. Finally, the two electrodes were put in contact with the sides of the box to get an effective gap distance of $35 \mathrm{~mm}$. Figure 2 presents a cross section of the final arrangement composed of the three electrode gap and the Plexiglas box containing the two non-mixing dielectric liquids.

During the experiment, three different dielectric liquids were considered:

- Glycerol, $\epsilon_{\mathrm{r}}=46$

- Silicone oil, $\epsilon_{\mathrm{r}}=2.2-2.9$

- Transformer oil, $\epsilon_{\mathrm{r}}=2.3$

Of these three materials, glycerol has the highest mass density and so will sink to the bottom if combined with either of the two other liquids. The electrical difference between the three dielectrics is that the permittivity (which is a bulk property), $\epsilon_{\mathrm{r}}$, of glycerol is substantially higher 
than the two others, as indicated above. The difference in dielectric constant between transformer oil and silicone oil is negligible. In the paper, we will refer to them as equal in magnitude each of them having the value $\epsilon_{\mathrm{r}}=3$. The silicone oil (AK350, supplied from Wacker Chemie $\mathrm{GmbH}$ ) was a polydimethylsiloxane (PDMS) fluid with viscosity $350 \mathrm{~mm}^{2} / \mathrm{s}$ and a number average of molar mass of the polymer, $M_{n}=14000 \mathrm{~g} / \mathrm{mol}$.

The measurements were performed in air under the following atmospheric conditions: temperature $25{ }^{\circ} \mathrm{C}$, pressure $101.1 \mathrm{kPa}$ and relative humidity $30 \%$. These parameters were constant during the measurements because the experiment was conducted in a climate controlled room. The starting point of every experiment was to fill the box with one liquid, record streamer currents for a given background field and needle voltage. Then, level by level the initial liquid was covered by an increasing layer of the second liquid (obviously, the same amount of the first liquid was removed before that). At each level, streamer data were recorded until the second liquid finally filled the box. In this way, we obtained the streamer currents at the needle and cathode and associated light signals from the propagating streamer as a function of the relative volume of the liquids. We conducted measurements for silicone oil combined with glycerol and transformer oil combined with glycerol. The reasons for choosing these two combinations was to change the liquid existing at the top and in that way observe possible differences due to surface properties.

\section{RESULTS OF MEASUREMENTS \\ 3.1 PRESENTATION OF A STREAMER SIGNAL AND COMPARISON OF STREAMER CURRENT IN AIR AND ALONG THE DIELECTRIC LIQUIDS}

Figure 3 shows one example from the performed measurements. It illustrates the simultaneous record of the needle and cathode currents for a streamer discharge

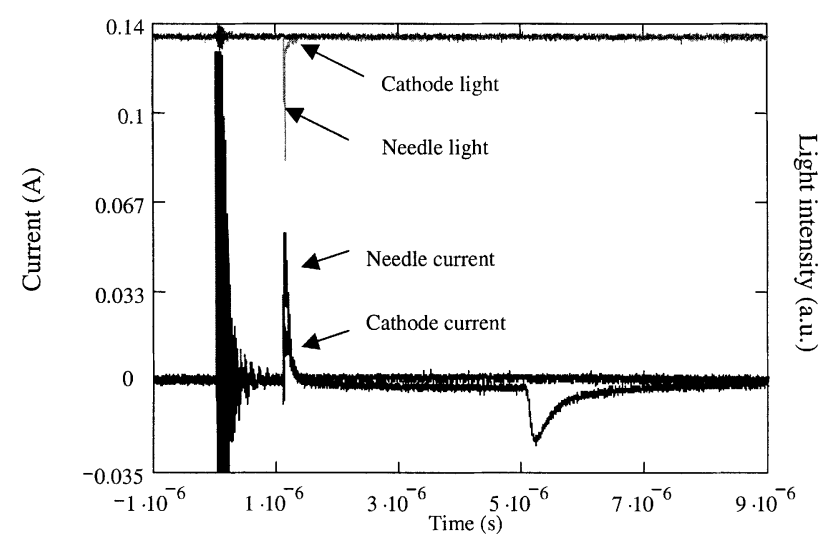

Figure 3. Simultaneous measurements of the needle and cathode current and light associated with a streamer propagating in air along glycerol in a $500 \mathrm{kV} / \mathrm{m}$ background field. event in the presence of only glycerol. The corresponding signals from the optical fibers placed at the needle and the cathode are also shown. The applied background field was $500 \mathrm{kV} / \mathrm{m}$ and the needle voltage was $3 \mathrm{kV}$. In the presented streamer signal, we notice the initial transients in the currents caused by the capacitive electromagnetic noise when the square voltage impulse is applied to the needle. The end of this square impulse is also visible in the needle current; it appears as a quite fast negative shift at time $5 \mu$ s after the start of the impulse. The streamer discharge event is the signal occurring at time $1.2 \mu \mathrm{s}$ after the application of the impulse on the needle. A corresponding light impulse is collected at the optical fiber placed at the needle. In our previous study [7], the current on the needle has been identified as a conduction current meanwhile the current at the cathode is a displacement current corresponding to the advancement of the positive streamer in the gap from the needle towards the cathode. By using the optical fiber placed at the cathode, one can determine if and when the streamer has arrived at the cathode. In Figure 3, there is almost no light detected at the fiber close to the cathode, meaning that in this particular case a very small amount of the light from the streamer has passed the fiber at that position. This would then mean that there is charge remaining or deposited in the gap, presumably at the surface of the glycerol.

From Figure 3, we can obtain the peak current at the needle and at the cathode respectively. These two quantities, especially the peak of the needle current, will be the main data taken from the streamer signals when analyzing the effect of the dielectric constant and the surface properties presented in section 3.2.

We can compare the current signals of streamers propagating in air alone and along two of the dielectric liquid surfaces, namely glycerol $\left(\epsilon_{\mathrm{r}}=46\right)$ and silicone oil $\left(\epsilon_{\mathrm{r}}=\right.$ 3). In Figure 4 is presented the current at the needle in the case of only glycerol and only silicone oil respectively. In the figure is also included the measurements with only air. Each signal corresponds to a different streamer dis-

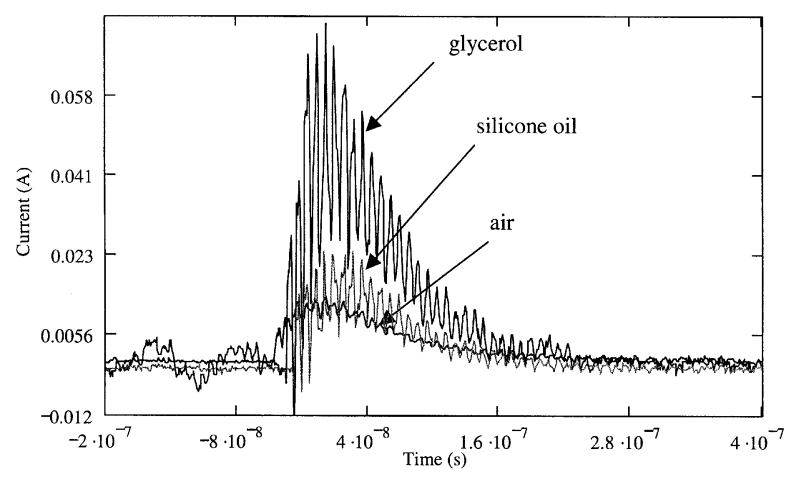

Figure 4. Comparison of the current measured at the needle for a streamer propagating in air, along the surface of glycerol and silicone oil respectively. The background field was $500 \mathrm{kV} / \mathrm{m}$ and the needle voltage was $3 \mathrm{kV}$. 
charge event. Again, the background field in each case was $500 \mathrm{kV} / \mathrm{m}$ and the needle voltage was $3 \mathrm{kV}$. The graph illustrates that the current magnitude at the needle is significantly larger for glycerol compared to silicone oil. Also, the current magnitude for silicone oil is somewhat larger compared to the case of air alone. Another interesting observation in the figure is the large oscillations present in the signal for the case of silicone oil and glycerol compared to the case of only air. Especially, one can see that the oscillations become larger for increasing magnitudes of the dielectric constant. One possible explanation could be the relatively larger capacitance of the electrode system when introducing the dielectrics in the gap.

\subsection{EFFECT OF THE DIELECTRIC CONSTANT AND SURFACE PROPERTIES}

The increase of the magnitude of the streamer current in the presence of a dielectric material compared to air, presented in section 3.1, indicates an increase of the ionization intensity. This increase of the ionization rate can be attributed to the field enhancement due to the relative permittivity of the dielectric but also to surface properties like the ionization potential of the surface, the presence of accumulated charge at the surface prior to streamer propagation, etc, as discussed in our previous study [6]. In this section we will use the experimental procedure explained in section 2 to study the separate contributions of the capacitive effect and surface properties. Two investigations will be performed, one in which the silicone oil is floating at the top and the glycerol is at the bottom and another in which the transformer oil is floating at the top meanwhile the glycerol is again located at the bottom. Section 3.2.1 presents the results of the first case meanwhile section 3.2.2 presents the second situation.

\subsubsection{SILICONE OIL FLOATING OVER GLYCEROL}

Figure 5 presents the magnitudes of the current peak at the needle as a function of the height (thickness) $\mathrm{H}$ (in $\mathrm{mm}$ ) of silicone oil floating over the glycerol in a background field of $500 \mathrm{kV} / \mathrm{m}$. The figure shows the results of 10 individual events performed for each particular height $\mathrm{H}$ of silicone oil. Note from Figure 5 that the measurements were performed only for the height $\mathrm{H}$ in the range 0-5 $\mathrm{mm}$ (corresponding to the volume $0-20 \mathrm{ml}$ of silicone oil). Only this range of heights $\mathrm{H}$ are interesting to analyze since the effect of the dielectric constant will be more pronounced with increasing amount of glycerol (which has the higher value of dielectric constant). From the figure, it is evident that there is a quite big dispersion of the data. The dispersion of the peak current is about $0.03 \mathrm{~A}$ with values ranging from 0.02 to $0.07 \mathrm{~A}$. In order to simplify analysis of the results, we have calculated for every level (or height $\mathrm{H}$ ) of silicone oil an average value of the peak current magnitudes. The average was formed us-

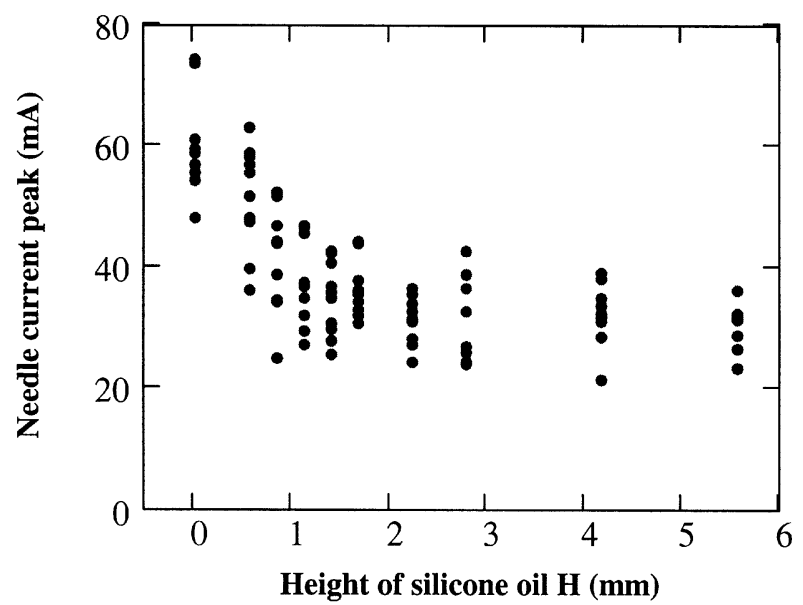

Figure 5. The streamer current peak $(\mathrm{mA})$ at the needle as a function of the height $\mathrm{H}(\mathrm{mm})$ of silicone oil floating above glycerol in a background field of $500 \mathrm{kV} / \mathrm{m}$. In the figure is shown the results of 10 individual events performed for each particular height $\mathrm{H}$ of silicone oil.

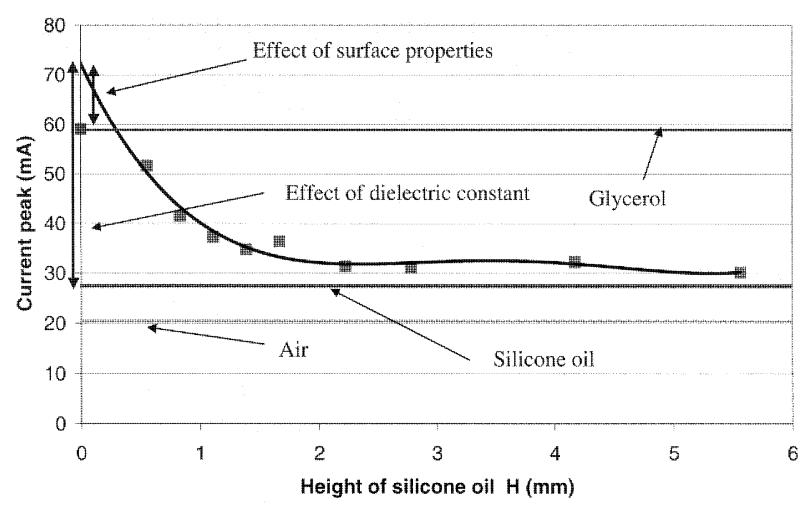

Figure 6. Polynomial curve fit of the average streamer current peak $(\mathrm{mA})$ at the needle as a function of the height $\mathrm{H}(\mathrm{mm})$ of silicone oil over the glycerol. The extrapolated point from the fitted curve at $\mathrm{H} \rightarrow 0$ is different than the actual measured point at $\mathrm{H}=0$.

ing the 10 individual events obtained at each particular silicone level. Figure 6 shows the corresponding results using the data presented in Figure 5.

We have also included in the figure the measured results of the streamer current in the case of only air, silicone oil and glycerol (solid lines in the figure at current magnitudes $0.0202 \mathrm{~A}, 0.0272 \mathrm{~A}$ and $0.0591 \mathrm{~A}$, respectively). Even these measurements are formed by taking the average of 10 events. The figure also shows a curve-fit of the averaged points for varying height $\mathrm{H}$ of the silicone oil using a polynomial function. The curve-fit has been made using all measured data points except the point corresponding to the glycerol surface, that is $\mathrm{H}=0$. Indeed, this is also the interesting fact that can be deduced from the measurements. By extending the calculated fit to the height $\mathrm{H}=0$ of silicone oil, the behavior of the glycerol and silicone oil combination can be investigated.

From Figure 6 we can see that when the amount of glycerol is increased, the streamer current magnitude in- 
creases more and more. However, since the surface properties of glycerol are not yet active, this increase must be only due to the dielectric constant of the glycerol. Indeed, what is going on here is that we are artificially changing the dielectric constant of the silicone oil from 3 to 46 when increasing the amount of glycerol. In the limit when the silicone oil volume is zero, that is $\mathrm{H}=0$, we can see from the extended fit that the increase in the streamer current due to the change in the dielectric constant from 3 to 46 would be about 2.6 times the value obtained for the silicone oil only (that is $0.0722 / 0.0272$ ). However, when we compare the measured point for the real glycerol surface with the extended fit at $\mathrm{H}=0$ we notice that there is a slight shift between these values. This means then that the real glycerol surface does not behave as the fictitious silicone oil material. Thus, the difference in current magnitude must be due to the effect of the glycerol surface properties compared to the silicone oil material. So we can see that we have quantified the capacitive contribution compared to the surface properties. The change in the current magnitude due to the increase of the dielectric constant is about $0.045 \mathrm{~A}$, meanwhile the change due to the effect of the surface properties is about $0.013 \mathrm{~A}$. Thus, the influence of the dielectric constant is about 3 times larger than the effect of the surface properties. Another interesting thing that can be observed from the graph is that the experimental point at $\mathrm{H}=0$ is shifted downwards compared to the point obtained from the fit. This would imply then that for the same dielectric constant, the streamer current is higher with the silicone oil surface properties than with the glycerol surface properties. It means that the silicone oil surface is aiding such that a higher streamer current is obtained compared to the glycerol surface, and this effect is only due to the surface properties.

\subsubsection{TRANSFORMER OIL FLOATING OVER GLYCEROL}

Figure 7 shows the averaged peak current amplitudes at the needle as a function of the height $\mathrm{H}$ (range 0-5 mm) of transformer oil floating over the glycerol in a background field of $500 \mathrm{kV} / \mathrm{m}$. Also here the average was formed using 10 individual events obtained at each particular transformer oil level. The average for only air, transformer oil and glycerol are also included in the figure each with values $0.0202 \mathrm{~A}, 0.0309 \mathrm{~A}$ and $0.0533 \mathrm{~A}$, respectively. Note, that there is a slight discrepancy in the measured value for the average current peak in the case of only glycerol shown in Figures 6 and 7. This is most probably due to the scattering of the data in the new sequence of measurements. In the figure is again included a polynomial curve fit of the results. As can be seen, we have more or less the same behavior as with the silicone oil floating over the glycerol. This time the increase in the streamer current due to the change in the dielectric constant from 3 to

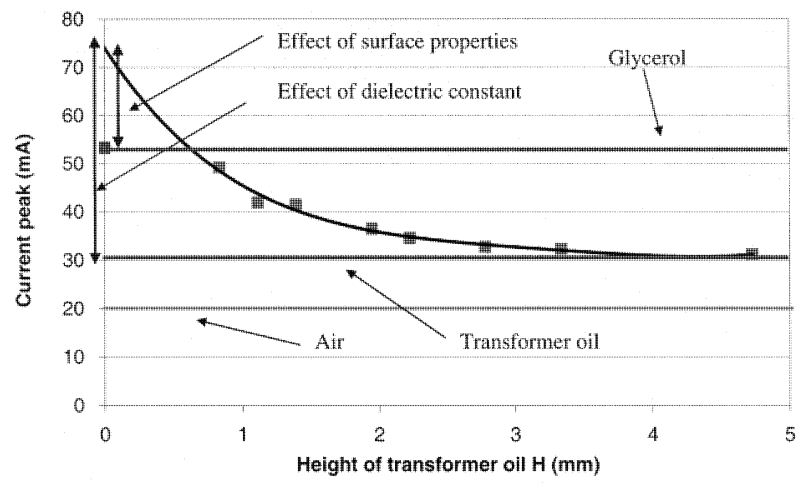

Figure 7. Polynomial curve fit of the average streamer current peak $(\mathrm{mA})$ at the needle as a function of the height $\mathrm{H}(\mathrm{mm})$ of transformer oil floating over the glycerol.

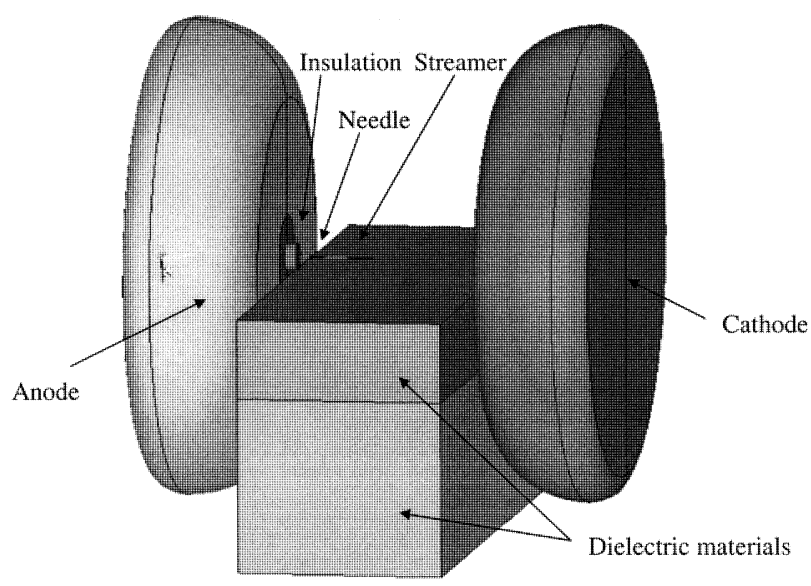

Figure 8. Illustration of the geometrical configuration used in the simulations.

46 is about 2.4 times the value obtained for the transformer oil only (that is $0.0741 / 0.0309$ ). If we neglect the discrepancy in the measured values for glycerol shown in Figures 6 and 7, we see that there is no big difference between the silicone oil surface and the transformer oil surface. They behave more or less the same. Especially, both of them seem to enhance more the streamer current compared to the glycerol surface properties.

\section{RESULTS OF SIMULATIONS}

We have used a 3-dimensional field calculation program (called ANSYS [9]), which is based on the finite element method to determine the electric field distribution in the three-electrode system in the presence of the two non-mixing dielectric materials. The purpose is to check whether we can reproduce numerically the observed dependence on the dielectric constant that we have in the measurements. Especially, we want to calculate the streamer charge (which basically corresponds to the time integral of the needle current) as a function of the relative amount of the two dielectric materials placed in the electrode gap. A very simplified model of the streamer propa- 
gating along the surface was used in the simulations. The streamer was represented as a cylindrically conducting channel which is in contact with the needle electrode. The streamer channel had a radius of $50 \mu \mathrm{m}$ and was terminated with a half sphere at its tip. This representation of the streamer might not be the most appropriate but is used because of its simplicity. In the simulations performed, we assumed that the conducting channel of the streamer was extending out $1 \mathrm{~cm}$ from the needle electrode. In the measurements, the insulator surface was placed $3 \mathrm{~mm}$ below the needle electrode. If we would assume that the streamer propagates straight, then the height of the streamer above the surface should be chosen as $3 \mathrm{~mm}$. However, this assumption of the streamer height might not be true because although the streamer is initiated from the needle tip it might be attracted to the insulator surface and eventually propagate on the surface itself [10]. In order to investigate the height dependence of the streamer, we have made simulations for two different heights above the insulator surface, $3 \mathrm{~mm}$ and $1 \mathrm{~mm}$, respectively. The insulator materials used in the simulations were assigned the dielectric constants $\epsilon_{\mathrm{r}}=3$ and $\epsilon_{\mathrm{r}}=$ 46 , respectively (where the first one was placed at the top). The background field was $500 \mathrm{kV} / \mathrm{m}$ and the needle was stressed with a constant voltage of magnitude $3 \mathrm{kV}$. Figure 7 shows a picture taken directly from the ANSYS-program showing in detail the geometrical configuration used, including the extending streamer channel. The height of the streamer above the surface is $3 \mathrm{~mm}$ in the illustrated case.

Figure 9 shows the charge in the streamer channel as a function of the height (thickness) $\mathrm{H}$ of silicone oil (or transformer oil) above the glycerol. The calculated values of the streamer charge have been divided with the magnitude when only the material with $\epsilon_{\mathrm{r}}=3$ is present. The results of the simulations for the two different streamer heights above the uppermost insulator surface $1 \mathrm{~mm}$ and $3 \mathrm{~mm}$ respectively are included in the figure. Note from

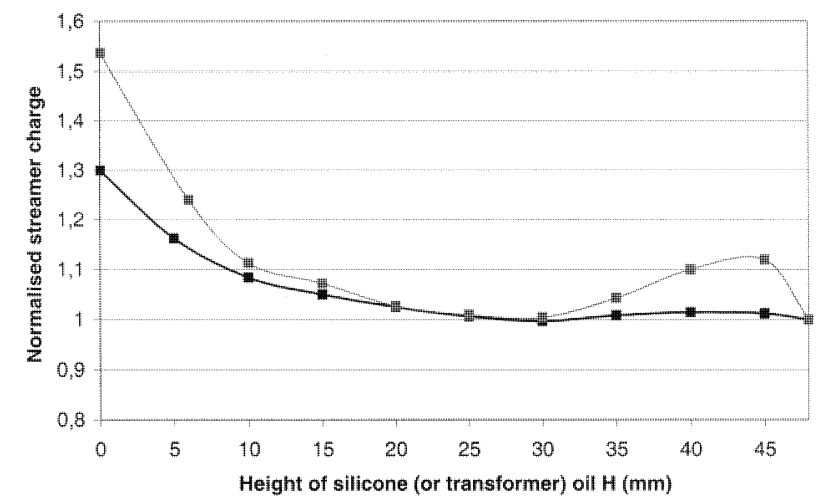

Figure 9. Results of simulations of the normalised streamer charge as a function of the height $\mathrm{H}$ of silicone (or transformer oil) above glycerol. Results are shown for two different heights $(1 \mathrm{~mm}$ and 3 $\mathrm{mm}$ respectively) of the streamer above the uppermost surface.
Figures 6 and 7 that the measurements were performed only for the height $\mathrm{H}$ in the range: $0-5 \mathrm{~mm}$ of silicone oil (or transformer oil), meanwhile the simulations shown in Figure 9 are performed for the whole range of heights $\mathrm{H}$ (from $0-48 \mathrm{~mm}$ ). The main conclusion that can be drawn from the diagram is that the capacitive behavior which we observed in the measurements when we increased the dielectric constant of the silicone oil (or transformer oil) is rather well reproduced in behavior (it is increasing when we increase the amount of glycerol) but not exactly in quantity. In the measurements we had a factor of about 2.5 times the value for only silicone oil or transformer oil when we increased the dielectric constant from 3 to 46 . According to the simulations, however, we observe only a factor of about 1.3 when we increase the dielectric constant from 3 to 46 . The second curve in the diagram shows the results when the streamer height is $1 \mathrm{~mm}$ above the insulator surface. Interestingly to see, we have now a higher contribution of the capacitive effect. This indicates therefore that when the streamer comes much closer to the insulator surface (it should literally propagate on the insulator), then the simulations would most probably attain a better quantitative agreement with the measurements.

\section{CONCLUSIONS}

$\mathrm{B}$ $Y$ measuring the currents of streamer discharges propagating in air and along different liquid dielectric surfaces, it has been possible to study the effects of the dielectric constant and the effect of the surface properties. First, it is clear that the presence of the dielectric surface increases the magnitude of the streamer current; this is mainly due to an enhancement of the electric field at the tip of the streamer because of the dielectric constant of the material which is higher than for the air. But, the main result of our study is the proof that there are also some surface properties of the material which affect the streamer. This was expected before but here we have been able to observe it experimentally. Especially we have quantified the contribution of each separate effect. Further, by using a simplified streamer model we could simulate with a 3D field calculation program the capacitive behavior and compare with the measurements. We found in general a good agreement between the measurements and the simulations. However, we discovered that the streamer height above the surface is an important factor. In order to quantitatively reproduce the measurements the streamer must propagate literally on the surface itself.

\section{ACKNOWLEDGEMENTS}

This work has been performed in the framework of the SSF-project ELIS. We would also like to thank Wacker Chemie, GmbH, for supply of the silicone oil (AK350). 


\section{REFERENCES}

[1] T. S. Sudarshan and R. A. Dougal, "Mechanism of Surface Flashover Along Solid Dielectrics in Compressed Gases: A Review”, IEEE Trans. Electr. Insul., Vol. 21, 727-746, 1986.

[2] A. Larsson, V. Cooray and V. Scuka, "Advanced Simulations of Space-charge Dominated Electrical Discharges in Air Along Dielectric Surfaces", EMB-98, Linköping, Sweden, 1998.

[3] L. Gao, Characteristics of Streamer Discharges in Air and along Insulating Surfaces, Doctoral Thesis, Institute of High Voltage Research, Uppsala University, 2000.

[4] N. L. Allen and A. Ghaffar, "Propagation of Positive Streamers Over Insulating Surfaces in Air", Conf. Electr. Insul. Dielectr. Phenomena (CEIDP), Paper 5B-14, 1995.

[5] N. L. Allen and P. N. Mikropoulos, "Streamer Popagation Along Insulating Surfaces", IEEE Trans. Dielectr. Electr. Insul., Vol. 6, p. 354, 1999.

[6] M. Akyuz, L. Gao, A. Larsson, V. Cooray, T. G. Gustavsson and S. M. Gubanski, "Positive Streamer Discharges along Insulating Surfaces", IEEE Trans. Dielectr. Electr. Insul., Vol. 8, pp. 902-910, 2001.

[7] M. Akyuz, L. Gao, A. Larsson and V. Cooray, "Streamer Current in a Three-electrode System", IEEE Trans. Dielectr. Electr. Insul., Vol. 8, pp. 665-672, 2001.

[8] L. Gao, M. Akyuz, A. Larsson, V. Cooray and V. Scuka, "Measurement of the Positive Streamer Charge", J. Phys. D: Appl. Phys., Vol. 33, p. 1861, 2000.

[9] ANSYS (Release 5.7), ANSYS Inc., Southpointe 275 Technology Drive Canonsburg, PA 15317, USA.

[10] M. Akyuz, A. Larsson, G. Strandberg and V. Cooray, "3D Simulations of Streamer Branching in Air”, J. Electrostatics, Vol. 59, pp. 115-141, 2003

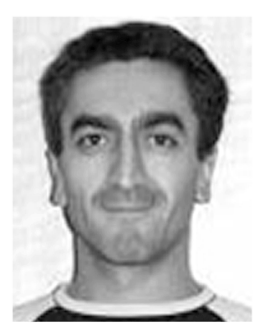

Mose Akyuz was born in Mydiat, Turkey in 1972. He received the M.Sc. degree in physics from Gothenburg University, Sweden, in 1997 and the Ph.D. degree in engineering science on atmospheric discharges from Uppsala University, Sweden, in 2002. Since 2003 he has worked as a scientist at the Swedish Defense Research Agency (FOI) mainly in the area of pulsed power.

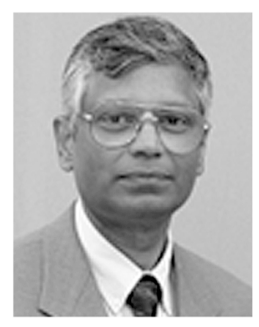

Vernon Cooray received the B.Sc. degree in Physics with first class honors from the University of Colombo Sri Lanka in 1975 and the Ph.D. degree in electricity with special attention to transients and discharges from the University of Uppsala, Sweden, in 1982. In 2000 he was promoted to the rank of full professor in electricity with special attention to transients and electrical discharges at the division for electricity and lightning research, Ångström Laboratory, University of Uppsala. Vernon Cooray has authored and co-authored more than 150 scientific papers in the physics of lightning and electrical discharges, lightning interaction and electromagnetic compatibility. He is the editor of the book The Lightning Flash published by IEE (London) in 2003 and the author of a popular book on lightning in Swedish Blixt och Åska så fungerar naturens fyreverkeri - published by Hallgren and Fallgren in 2003. Vernon Cooray is a member of the Scientific Committee of the International Conference on Lightning Protection. 